\title{
Innovar en la asignatura de Prevención de Riesgos Laborales: ¿una cuestión de riesgos o de protección de la enseñanza?
}

\author{
Ana Domínguez MORALES \\ Universidad de Sevilla \\ Departamento de Derecho del Trabajo \\ y de la Seguridad Social \\ admorales@us.es \\ D.0.I.: http://dx.doi.org/10.12795/JDU.2018.i01.11 \\ Pp.: 198-215
}

\section{Resumen}

La tarea de innovación docente se lleva a cabo en la asignatura de Prevención de Riesgos Laborales, impartida en el Doble Grado de Relaciones Laborales, Recursos Humanos y Finanzas y Contabilidad de la Facultad de Ciencias del Trabajo. La metodología empleada se basa en la problematización y reelaboración de ideas, enfoque que enlaza con el modelo de investigación por parte del alumno contando con el impulso y guía de la profesora. De ese modo, el estudiante debe participar en debates, reflexionar sobre asuntos propuestos y realizar actividades tanto individuales como grupales que posteriormente pueden ser expuestas ante a sus compañeros. Los resultados demuestran la necesidad de un replanteamiento general de la docencia que se centre en la necesaria participación activa del alumno. 
Palabras Clave: Prevención de Riesgos Laborales, Doble Grado en Relaciones Laborales, Recursos Humanos y Finanzas y Contabilidad, Docencia Universitaria, Renovación Docente Universitaria.

\section{Marco contextual}

El ciclo de mejora se lleva a cabo en la asignatura de Prevención de Riesgos Laborales, una asignatura de notable peso y relevancia en el Departamento de Derecho del Trabajo y de la Seguridad Social desde la modificación de los planes de estudio introducida por la Ley de Reforma Universitaria que asumía, a su vez, las exigencias del Espacio Europeo de Enseñanza Superior (Rodríguez-Piñero Royo, 2012). En concreto, se ha impartido en el Doble Grado de Relaciones Laborales, Recursos Humanos y Finanzas y Contabilidad de la Facultad de Ciencias del Trabajo. Es una asignatura de carácter anual, de manera que los alumnos ya estaban familiarizados con los conceptos más áridos de la misma, de un elevado nivel técnico, lo que permitiría no detenernos en conceptos básicos y llevar a cabo otro tipo de actividades no programadas antes.

Concretamente se utilizó el tema correspondiente a la responsabilidad del empresario y de otros sujetos obligados por las normas de prevención de riesgos laborales ante el incumplimiento de las mismas. Al ser un aspecto muy concreto de la asignatura y delimitable en dos subtemáticas, se decidió utilizarlo para llevar a cabo los dos ciclos de mejora desarrollados durante el Curso General de Docencia Universitaria. En el primero de ellos se abordarían dos de las cuatro responsabilidades oponibles ante la omisión de la correcta aplicación de las normas sobre seguridad y salud de los trabajadores: responsabilidad administrativa (multas económicas) y penal (delitos generales y específicos); en el segundo ciclo de mejora nos centramos en las dos restantes: responsabilidad en el ámbito de la Seguridad Social (recargo de las prestaciones) y Civil (indemnización de daños y perjuicios). 


\section{Diseño del Ciclo de Mejora Docente}

\section{Mapa de contenidos}

El esquema o mapa desde el que parte nuestro diseño del Ciclo de Mejora (Figuras 1 y 2) contempla los aspectos conceptuales y procedimentales -integrando el contenido actitudinal-que se han tenido en cuenta para la previsión de las actividades que se realizaron en las cuatro sesiones en las que se desarrolla el ciclo. El mismo se dedicaría a la responsabilidad en materia de Seguridad Social y Civil en casos de incumplimiento de las normas de prevención de riesgos laborales cuando el trabajador resulta dañado o fallece por circunstancias laborales.

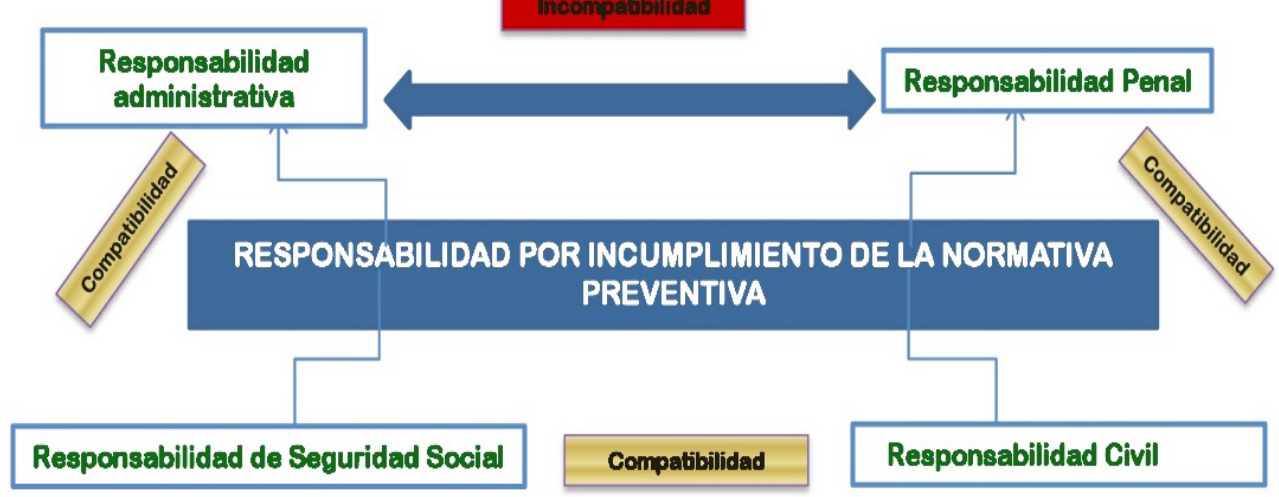

Figura 1: Mapa de contenidos. Problemáticas centrales tratadas en los dos ciclos de mejora.

Jornadas de Formación e Innovación Docente del Profesorado | № 1 (2018)

(i) Esta obra se distribuye con la licencia Creative Commons Reconocimiento-NoComercial-SinObraDerivada $\quad 4.0$ Internacional (CC BY-NC-ND 4.0.) 
Actividad 3:

Busqueda de sentencias sobre

distintos

sumuestrs en

los que se

solicita la

imposición

del recargo

Aclividad 4.

Expusiciones

urales de la

aclividad 3 y

comenlarius

Leyenda:

-C. Concept.ral

-O. Procedinent

Antivirlarles

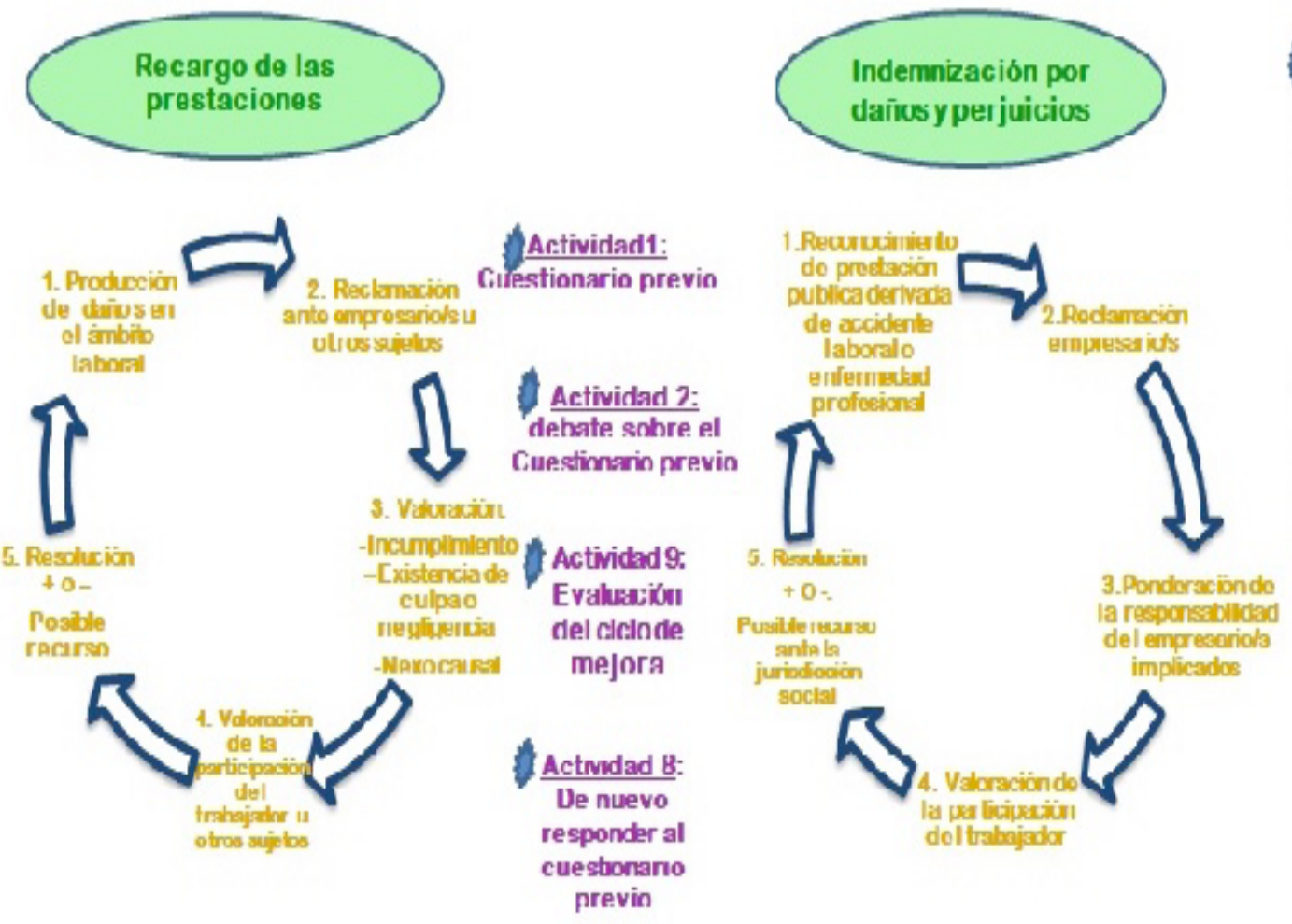

c.ividar $5:$

royecciondeun video sobre las

modificaciones de la ley que regula - l baromo do

indermizaciones

Actividad 6 Plantozrionto do supuesting anios que se soliclta

inckemui<ación por daños y perjucicios con distıntas

I esuluciones

Activictad 7: Exposiciones oralee dela actividad $6 y$ comentarioe

Figura 2. Mapa de contenidos del segundo ciclo de mejora.

Jornadas de Formación e Innovación Docente del Profesorado | № 1 (2018)

(c) (i) $\odot$ Esta obra se distribuye con la licencia Creative Commons

Internacional (CC BY-NC-ND 4.0.) 
En primer lugar, la responsabilidad en materia de Seguridad Social se traduce en términos materiales en la imposición al empresario de un recargo de las prestaciones públicas reconocidas al trabajador que sufre un accidente laboral o contrae una enfermedad profesional o de las prestaciones por viudedad $u$ orfandad reconocidas a los causahabientes del trabajador que fallece como consecuencia de la omisión del deber de cumplir con las normas preventivas. Este recargo supone una sanción económica calculada sobre el importe de la prestación pública.

Por su parte, la responsabilidad civil desencadena la imposición a los sujetos responsables del pago de la denominada indemnización de daños y perjuicios a los trabajadores que han sufrido un daño o han contraído una enfermedad en el trabajo habida cuenta del incumplimiento de las normas protectoras de seguridad y salud. En caso de fallecimiento la indemnización puede ser solicitada y reconocida a sus causahabientes.

\section{Modelo metodológico posible y secuencia de actividades programadas}

A partir de la bibliografía consultada, consideramos oportuno desarrollar un modelo metodológico (Figura 3) basado en la problematización de las ideas (Finkel, 2008), y por tanto de carácter investigativo. Así pues, se trataba de que el alumno pudiera experimentar mayor interés en el problema que se le planteaba, al tener que ir descubriendo por sí mismo el sentido de la actividad, lo que permitía conseguir un aprendizaje a largo plazo (Mora, 2017).

\section{$P R->I E->$ AC $\rightarrow C->$ A}

Pr: Problematización. le: Ideas de los alumnos.

Ac: Actividad de contraste. C: Conclusiones. A: Autoevaluación.

Figura 3. Modelo metodológico.

Jornadas de Formación e Innovación Docente del Profesorado I № 1 (2018) Esta obra se distribuye con la licencia Creative Commons 
Para desarrollar el ciclo se realizaron 9 actividades (Tabla 1), incluyendo la aplicación de un cuestionario inicial y final relacionado con el objeto de estudio, así como otras actividades que permitirían a los alumnos entrar en contacto con la realidad de lo que se trataba de enseñar (documentos jurídicos, vídeos explicativos o redacción de sentencias).

Tabla 1. Secuencia de actividades del ciclo de mejora.

\begin{tabular}{|c|c|c|c|}
\hline Actividad & Sesión & Contenido & Duración \\
\hline 1 & $\begin{array}{l}\text { Primera. } 3 \text { de mayo. } \\
\text { Introducción. }\end{array}$ & $\begin{array}{l}\text { Cuestionario previo so- } \\
\text { bre problemas clave de } \\
\text { la materia que se va a } \\
\text { abordar, utilizando pre- } \\
\text { guntas abiertas, indi- } \\
\text { rectas y con un enfoque } \\
\text { práctico. }\end{array}$ & 15 minutos. \\
\hline 2 & $\begin{array}{l}\text { Primera. } 3 \text { de mayo. } \\
\text { Introducción. }\end{array}$ & $\begin{array}{l}\text { Debate sobre el conte- } \\
\text { nido del cuestionario } \\
\text { inicial. }\end{array}$ & 15 minutos. \\
\hline 3 & $\begin{array}{l}\text { Primera. } 3 \text { de mayo. } \\
\text { Responsabilidad en } \\
\text { materia de Seguri- } \\
\text { dad Social (recargo } \\
\text { de prestaciones). }\end{array}$ & $\begin{array}{l}\text { Búsqueda, por parejas, } \\
\text { de una sentencia que re- } \\
\text { suelva la reclamación } \\
\text { del recargo de prestacio- } \\
\text { nes ante el posible in- } \\
\text { cumplimiento de las } \\
\text { normas preventivas. La } \\
\text { profesora les asigna su- } \\
\text { puestos determinados } \\
\text { que debían encontrar. }\end{array}$ & $\begin{array}{l}80 \text { minu- } \\
\text { tos (más tra- } \\
\text { bajo en casa } \\
\text { de algunas } \\
\text { parejas). }\end{array}$ \\
\hline 4 & $\begin{array}{l}\text { Segunda. } 4 \text { de mayo. } \\
\text { Responsabilidad en } \\
\text { materia de Seguri- } \\
\text { dad Social (recargo } \\
\text { de prestaciones). }\end{array}$ & $\begin{array}{l}\text { Exposiciones orales de la } \\
\text { actividad } 3 \text { y debate. }\end{array}$ & 105 minutos. \\
\hline
\end{tabular}

Jornadas de Formación e Innovación Docente del Profesorado | № 1 (2018) Esta obra se distribuye con la licencia Creative Commons Reconocimiento-NoComercial-SinObraDerivada Internacional (CC BY-NC-ND 4.0.) 


\begin{tabular}{|c|c|c|c|}
\hline 5 & $\begin{array}{l}\text { Tercera. } 10 \text { de mayo. } \\
\text { Responsabilidad civil } \\
\text { (indemnización de } \\
\text { daños y perjuicios). }\end{array}$ & $\begin{array}{l}\text { Proyección de un vídeo } \\
\text { explicativo sobre la re- } \\
\text { forma que aprueba el } \\
\text { nuevo baremo de ac- } \\
\text { cidentes de vehículos } \\
\text { a motor aplicado por } \\
\text { los jueces de la juris- } \\
\text { dicción social de modo } \\
\text { orientativo para cuanti- } \\
\text { ficar los daños a la inte- } \\
\text { gridad física que sufren } \\
\text { los trabajadores como } \\
\text { consecuencia de un ac- } \\
\text { cidente laboral o en- } \\
\text { fermedad profesional. } \\
\text { Posterior comentario so- } \\
\text { bre el mismo. }\end{array}$ & 10 minutos. \\
\hline 6 & $\begin{array}{l}\text { Tercera. } 10 \text { de mayo. } \\
\text { Responsabilidad civil } \\
\text { (indemnización por } \\
\text { daños y perjuicios). }\end{array}$ & $\begin{array}{l}\text { Descripción de un proce- } \\
\text { dimiento judicial de pe- } \\
\text { tición de indemnización } \\
\text { por daños y perjuicios } \\
\text { con distintas resolucio- } \\
\text { nes: admisión o denega- } \\
\text { ción de la reclamación. } \\
\text { Se utilizan supuestos de } \\
\text { hecho dados por la pro- } \\
\text { fesora y se trabaja por } \\
\text { parejas. }\end{array}$ & $\begin{array}{l}95 \text { minu- } \\
\text { tos (más tra- } \\
\text { bajo en casa } \\
\text { de algunas } \\
\text { parejas). }\end{array}$ \\
\hline 7 & $\begin{array}{l}\text { Cuarta.11 de mayo. } \\
\text { Responsabilidad civil } \\
\text { (indemnización de } \\
\text { daños y perjuicios). }\end{array}$ & $\begin{array}{l}\text { Exposiciones orales de la } \\
\text { actividad } 6 \text { y debate. }\end{array}$ & 95 minutos. \\
\hline 8 & $\begin{array}{l}\text { Cuarta.11 de mayo. } \\
\text { Cierre del ciclo. }\end{array}$ & $\begin{array}{l}\text { Resolución del cuestio- } \\
\text { nario final, coincidente } \\
\text { con el inicial. }\end{array}$ & 15 minutos. \\
\hline 9 & $\begin{array}{l}\text { Posterior al ciclo. } \\
\text { Cierre del ciclo. }\end{array}$ & $\begin{array}{l}\text { Cuestionario de autoe- } \\
\text { valuación del ciclo de } \\
\text { mejora. }\end{array}$ & $\begin{array}{l}\text { Fuera de } \\
\text { clase. }\end{array}$ \\
\hline
\end{tabular}

Completada la secuencia de actividades, se realiza una evaluación tanto de la evolución del aprendizaje de los alumnos como del propio ciclo de mejora, como se verá a continuación. 


\section{Cuestionario inicial-final}

Al inicio del ciclo, los alumnos debían contestar de forma individual, nominativa y por escrito a un cuestionario en el que se hacian preguntas abiertas e indirectas y con un enfoque práctico sobre asuntos clave de la materia que íbamos a abordar durante el mismo.

Tal actividad se realizó con la idea de servir de instrumento de evaluación del conocimiento previo que poseían los alumnos sobre las problemáticas que iban ser desarrolladas durante la ejecución del ciclo de mejora. De este modo, se trataba de poner en evidencia las carencias iniciales que podían suponer mayores dificultades para la consecución de las actividades planificadas. Al mismo tiempo, se usaría como herramienta de comparación entre lo que el alumno ya conocía inicialmente y lo que habría aprendido una vez finalizado el ciclo.

Para evaluar los resultados, se ha realizado una escalera de aprendizaje que coloca en cada escalón los grupos de respuestas en función de la profundidad o acierto de la misma de forma ascendente (Rivero y Porlán, 2017). A través de ella se han podido destacar los obstáculos a los que se enfrentan los alumnos para subir de peldaño.

\section{Aplicación del Ciclo de Mejora Docente}

\section{Relato resumido de las sesiones}

Los alumnos, en general, se mostraron, en principio, reacios a que el grueso de las sesiones se dedicase al trabajo propio, individual o en grupo. Fueron bastante parcos en sus respuestas al cuestionario inicial. Tampoco fue muy participativo el debate que siguió el cuestionario a pesar de que la profesora trató de avivarlo. 
La búsqueda de sentencias sobre supuestos concretos de imputación o no del recargo de las prestaciones supuso un importante reto para ellos porque no estaban familiarizados con instrumentos jurídicos como las sentencias y mucho menos con la búsqueda de las mismas en bases de datos. Esta circunstancia motivó la reflexión de la profesora sobre la falta de aprendizaje previo de aspectos absolutamente esenciales o básicos para alumnos de tercer curso en el grado en Relaciones Laborales, Recursos Humanos y Finanzas y Contabilidad. Siendo consciente de tal situación, se les mostraron las herramientas necesarias para comenzar a trabajar. Esencialmente portales informáticos donde podrían buscar y encontrar de una forma sencilla sentencias, además de explicarles - de nuevo- la estructura de las mismas. Habiendo finalizado su trabajo de forma exitosa, las exposiciones orales se realizaron con gran soltura si bien los estudiantes fueron, en su mayoría, poco participativos en el debate, a pesar de que la profesora trató de hacer preguntas directas a los alumnos.

Tampoco fue sencillo hacerles comprender cómo los jueces de la jurisdicción social calculaban la indemnización de daños y perjuicios derivada de accidente laboral o enfermedad profesional. Se les mostró un video explicativo que debió ser aclarado en sus términos conceptuales básicos por la profesora.

Posteriormente, los alumnos debían imaginar y redactar una sentencia que contuviera los hechos probados, los argumentos jurídicos y el fallo de un supuesto de hecho que la profesora les asignó que daba como resultado la imputación de la indemnización de daños y perjuicios o la denegación de la misma. Al principio, surgió de nuevo un clima de inseguridad y un sentimiento de incapacidad inicial para realizar el trabajo propuesto, preocupante para la profesora, problema que pronto fue solventado con algunas ideas que iba señalando la misma a quienes no lograban imaginar el supuesto que se le había asignado. Finalmente mostraron haber comprendido su cometido a 
través de las exposiciones. El debate que siguió a tal actividad fue parco, de nuevo, demostrándose una vez más que es necesario que el alumno participe activamente desde el inicio de su formación académica para evitar que en los cursos superiores encontremos estudiantes pasivos y poco entusiastas con las materias objeto de estudio.

Cerramos el ciclo con el cuestionario final al que contestaron de forma algo más concisa que al inicio del ciclo, aunque demostraron en algunos casos falta de aprendizaje, siendo esto último algo desconcertante pues se apreciaba en sus exposiciones haber aprehendido los distintos conceptos y procedimientos sobre los que debían trabajar. Algo más extensas fueron las observaciones realizadas a la autoevaluación que se les remitió vía plataforma universitaria, comentarios que se asumen como algo positivo y que sirven a la profesora para adoptar algunas mejoras en el método docente en el futuro.

\section{Evaluación del aprendizaje de los estudiantes}

El cuestionario que los estudiantes completaron al inicio y al final del ciclo se conformaba por seis preguntas guiadas por un hilo conductor o supuesto de hecho.

Las preguntas fueron las siguientes:

1. Lola, trabajadora de Jamonarte, contratista de Iberriquísimo, ha sufrido un accidente manipulando una máquina cortadora. Dicha herramienta no disponía del dispositivo de seguridad. La trabajadora acude al hospital y automáticamente cursan su baja laboral. ¿Tiene derecho a una prestación pública?

2. Si demuestra que el accidente no se habría producido de mediar el sistema de seguridad o utilizar un Equipo de Protección Individual, ¿podría incrementarse esta prestación pública? 
3. Si la respuesta anterior fue positiva ¿quién o quiénes serian sujetos responsables del pago del mismo?

4. Aparte de la responsabilidad penal, administrativa o de Seguridad social, ¿existe algún otro ámbito del ordenamiento jurídico que pueda servir a Lola para reclamar una indemnización por el daño sufrido?, ¿cuál?

5. Si has contestado afirmativamente a la pregunta 2, ¿crees que sería compatible la responsabilidad en materia de Seguridad Social con una posible sanción pecuniaria por incumplimiento de las normas preventivas?

6. En su caso, ¿a qué empresario podría reclamar dicha indemnización?, ¿es posible que exista algún otro sujeto a quien reclamar el pago de la misma?

Para poder llevar a cabo un ejercicio de investigación sobre la evolución del aprendizaje las respuestas a cada una de las preguntas se dividieron en escalones en atención al mayor acierto y concreción de las mismas (Tabla 2).

Tabla 2. Escalones de conocimiento en las respuestas al cuestionario.

\begin{tabular}{|l|l|}
\hline Pregunta 1 & Respuestas \\
\hline Escalón 0 & Respuesta incorrecta \\
\hline Escalón 1 & Contesta sí \\
\hline Escalón 2 & Contesta sí, por accidente de trabajo \\
\hline Escalón 3 & $\begin{array}{l}\text { Contesta sí, por incapacidad temporal aunque razona } \\
\text { incorrectamente }\end{array}$ \\
\hline Escalón 4 & Contesta sí, por incapacidad temporal sin razonar \\
\hline Escalón 5 & $\begin{array}{l}\text { Contesta sí, por incapacidad temporal razonando que de- } \\
\text { riva de accidente laboral }\end{array}$ \\
\hline Escalón 6 & $\begin{array}{l}\text { Contesta sí, por incapacidad temporal razonando que de- } \\
\text { riva de accidente laboral }\end{array}$ \\
\hline Pregunta 2 & Respuestas \\
\hline
\end{tabular}

Jornadas de Formación e Innovación Docente del Profesorado I № 1 (2018) Esta obra se distribuye con la licencia Creative Commons Reconocimiento-NoComercial-SinObraDerivada Internacional (CC BY-NC-ND 4.0.) 


\begin{tabular}{|c|c|}
\hline Escalón 0 & Respuesta incorrecta \\
\hline Escalón 1 & Contesta sí \\
\hline Escalón 2 & Contesta sí, pero razona la respuesta incorrectamente \\
\hline Escalón 3 & Contesta si y razona la respuesta correctamente \\
\hline Escalón 4 & $\begin{array}{l}\text { Contesta sí, indicando que a eso se denomina recargo de } \\
\text { prestaciones }\end{array}$ \\
\hline Escalón 5 & $\begin{array}{l}\text { Contesta sí, señalando el recargo de prestaciones y añade } \\
\text { información correcta que no se había preguntado }\end{array}$ \\
\hline Pregunta 3 & Respuestas \\
\hline Escalón 0 & Respuesta incorrecta \\
\hline Escalón 1 & $\begin{array}{l}\text { Señala a un posible sujeto responsable, el empresario, } \\
\text { pero añade otros de forma incorrecta }\end{array}$ \\
\hline Escalón 2 & Señala a un posible sujeto responsable, el empresario \\
\hline Escalón 3 & $\begin{array}{l}\text { Señala a dos posibles sujetos responsables, empresa- } \\
\text { rio principal y empresario contratista, pero señala otro } \\
\text { incorrectamente }\end{array}$ \\
\hline Escalón 4 & $\begin{array}{l}\text { Contesta sí, indicando que a eso se denomina recargo de } \\
\text { prestaciones }\end{array}$ \\
\hline Pregunta 4 & Respuestas \\
\hline Escalón 0 & Respuesta incorrecta \\
\hline Escalón 1 & Contesta sí \\
\hline Escalón 2 & $\begin{array}{l}\text { Contesta sí, pero señala un ámbito del ordenamiento } \\
\text { incorrecto }\end{array}$ \\
\hline Escalón 3 & $\begin{array}{l}\text { Contesta sí, señalando un ámbito del ordenamiento co- } \\
\text { rrecto y otro incorrecto }\end{array}$ \\
\hline Escalón 4 & $\begin{array}{l}\text { Contesta si, señalando el ámbito del ordenamiento } \\
\text { correcto }\end{array}$ \\
\hline Pregunta 5 & Respuestas \\
\hline Escalón 0 & Respuesta incorrecta \\
\hline Escalón 1 & Contesta sí \\
\hline Escalón 2 & Contesta sí, sí pero añade razonamiento incorrecto \\
\hline Escalón 3 & $\begin{array}{l}\text { Contesta sí, pero añade razonamiento ulterior no pedido } \\
\text { pero correcto }\end{array}$ \\
\hline Pregunta 6 & Respuestas \\
\hline Escalón 0 & Respuesta incorrecta \\
\hline
\end{tabular}

Jornadas de Formación e Innovación Docente del Profesorado | № 1 (2018) Esta obra se distribuye con la licencia Creative Commons Reconocimiento-NoComercial-SinObraDerivada $\quad 4.0$ Internacional (CC BY-NC-ND 4.0.) 


\begin{tabular}{|l|l|}
\hline Escalón 1 & Solo señala a un posible sujeto responsable \\
\hline Escalón 2 & Señala a dos posibles sujetos responsables \\
\hline Escalón 3 & Señala a tres posibles sujetos responsables \\
\hline Escalón 4 & $\begin{array}{l}\text { Señala a más de tres posibles sujetos responsables } \\
\text { siendo alguno incorrecto }\end{array}$ \\
\hline Escalón 5 & Señala a más de tres posibles sujetos responsables \\
\hline
\end{tabular}

Como puede observarse en los gráficos 1 al 6, se aprecia en general cierto avance de los primeros escalones a los siguientes, según se evidencia claramente en las respuestas a la segunda y sexta preguntas. Si bien, no se manifiesta una subida en bloque o muy significativa hacia los niveles más altos, salvo en la pregunta cuarta. En muchos casos se mantienen los mismos niveles en ambas respuestas a pesar de haber finalizado el ciclo, como demuestran los resultados de las preguntas uno y cinco. No obstante, aunque se puede apreciar que prácticamente ninguno se sitúa al cerrar el ciclo en el escalón cero, resulta llamativa la presencia de un porcentaje -reducido- de alumnos que se colocan en ese nivel en las preguntas 3 y 5 , al tiempo que se observa que no se sitúan en tal escalón ni al inicio ni al final del ciclo ninguno de los participantes en relación a las preguntas 1 y 6 .

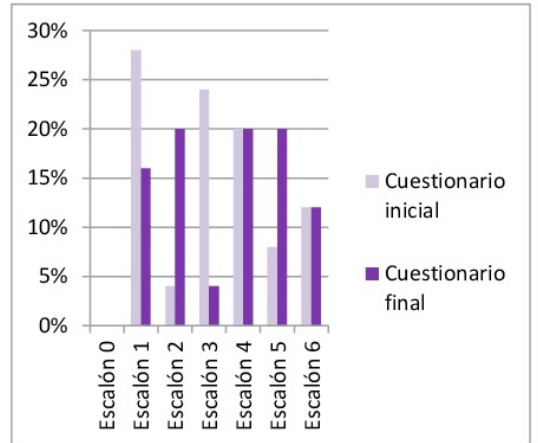

Gráfico 1. Pregunta 1. Respuestas a los cuestionarios inicial y final

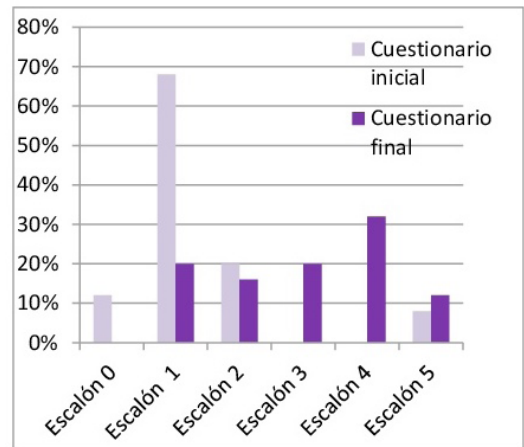

Gráfico 2. Pregunta 2. Respuestas a los cuestionarios inicial y final. 


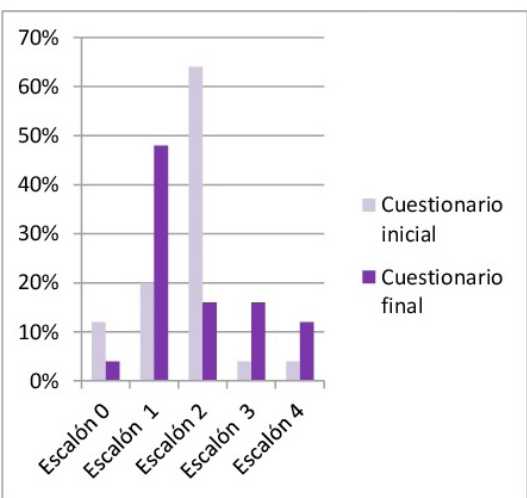

Gráfico 3. Pregunta 3. Respuestas a los cuestionarios inicial y final

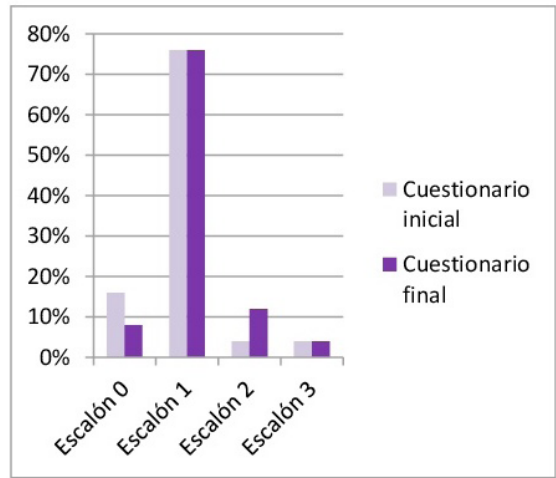

Gráfico 5. Pregunta 5. Respuestasa los cuestionarios inicial y final.
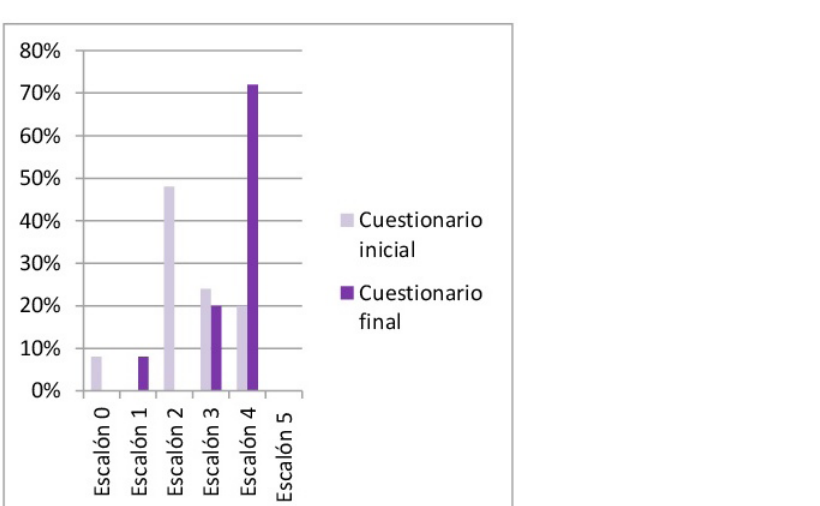

Gráfico 4. Pregunta4. Respuestas a los cuestionarios inicial y final.

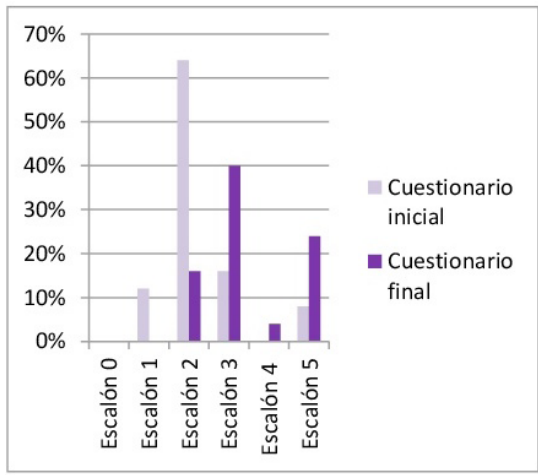

Gráfico 6. Pregunta6. Respuestas a los cuestionarios inicial y final

\section{Evaluación del Ciclo de Mejora Docente}

\section{Evaluación de la participación de los alumnos}

La evolución del aprendizaje de los alumnos ha sido relativa, como demuestran los porcentajes obtenidos en los distintos escalones de aprendizaje, que muestran un número reducido de estudiantes que han progresado hasta los niveles superiores. No obstante, la experiencia ha sido muy satisfactoria en general tanto para la profesora como 
para ellos mismos, tal y como han expresado una vez finalizado el ciclo de mejora en un cuestionario de autoevaluación que se les entregó a través de la plataforma virtual.

Se valora positivamente la alta asistencia de los alumnos y su participación en todas las sesiones, aunque hay que señalar como aspecto negativo su menor predisposición a debatir, si bien admiten que poder dar su opinión en clase sobre las materias que se trabajan resulta necesario.

En su trabajo en parejas o grupos fueron más comunicativos y resaltó el compañerismo. A medida que fue avanzando el ciclo adoptaron una actitud proactiva y de mayor concentración, cuestiones que también se valoran de forma positiva por parte de la profesora.

\section{Evaluación de la docencia}

El seguimiento del curso de formación docente y el desarrollo de los ciclos de mejora han supuesto el descubrimiento de nuevas estrategias y técnicas de enseñanza que antes no se habían puesto en práctica, como es la de comenzar el análisis de un tema concreto partiendo de la problematización de las ideas. Consideramos muy eficaz comenzar las lecciones con el planteamiento de un problema de partida, ya sea a través de un caso concreto o de distintas preguntas de cuya respuesta parta un debate con el que se dé pie a la reflexión en grupo y así se despierte el interés del alumno por conocer el resultado de la cuestión central.

Además, se ha podido advertir que es posible dar clase con la boca "parcialmente cerrada", asumiendo así la propuesta de Finkel (2008), pues, a pesar del título de su obra, el autor atribuye al profesor un papel de guía o mediador entre el problema y los alumnos. Una vez que los estudiantes se encuentran suficientemente motivados, son capaces de utilizar por sí mismos las herramientas de trabajo 
que encuentran a su disposición para resolver los problemas planteados.

Se pone en evidencia que el profesor siempre debe estar presente para los alumnos, para proponer ideas de las que carecen en un primer momento, o para explicar determinados conceptos o aportar matices a sus trabajos. De lo contrario, los resultados pueden no ser los esperados porque, si no asumen ciertas pautas iniciales, los estudiantes tienden a encerrarse en sí mismos y considerarse incapaces de tomar las riendas en una materia totalmente desconocida por ellos.

Por todo lo anterior y en aras de una mejora de la calidad docente, se estima la aplicación para el futuro de los siguientes principios didácticos y herramientas de enseñanza:

- Consideramos como principio rector o básico en una materia docente hacer del alumno el centro de cada sesión, fomentando la reflexión individual y la pérdida del miedo a compartir sus ideas, inquietudes o cuestiones con el resto de compañeros y con el profesor.

- Siempre teniendo presente lo anterior, consideramos adecuado el método de la problematización de las ideas seguido de breves exposiciones conceptuales que aporten al alumno el conocimiento necesario para trabajar por ellos mismos sobre supuestos prácticos. No obstante, no es necesario que cada una de las sesiones deba comenzary desarrollarse del mismo modo, pues en ocasiones una lección de contenido eminentemente teórico-descriptivo también puede ser muy útil. Se trata, por tanto, de intercalar las actividades de investigación, de trabajo en grupo y de exposición pública de sus trabajos con las explicaciones teóricas del profesor, sin que se deba mantener siempre el mismo esquema para todas las sesiones y en todas las asignaturas (Bain, 2004). De esta forma podemos romper con lo que se ha denominado "la estructura básica del aula tradicional” (Fernández Enguita, 2018). 
- Se debe evitar la repetición de las mismas actividades, tratando de innovar continuamente, sin repetir modelos de actividades y exposiciones orales del trabajo realizado de forma sistemática para no perder la atención de quienes no están hablando en público. Para evitarlo, es preciso hacer partícipe también a los alumnos que atienden pasivos a tales exposiciones, por ejemplo, exigiendo un comentario o pregunta posterior de estos al alumno que expone o bien directamente preguntando a los oyentes sobre lo que acaban de escuchar.

- En la medida en que así lo permita el proyecto docente de la asignatura, creemos oportuno introducir un sistema de evaluación que tenga más en cuenta el trabajo del alumno, incluyendo las actividades realizadas, su comportamiento, la fluidez oral de sus intervenciones, el compañerismo o la simple presencia en clase.

- Finalmente, se considera positivo introducir sistemas de autoevaluación del profesorado durante el curso y no solo a través del sistema unitario o global realizado por la Universidad. De este modo, en el transcurso del año académico se puede adaptar la metodología docente al curso concreto. 


\section{Referencias bibliográficas}

Bain, K. (2004). Lo que hacen los mejores profesores universitarios. Valencia: Publicaciones de la Universidad de Valencia.

Finkel, D. (2008). Dar clase con la boca cerrada. Valencia: Publicaciones de la Universidad de Valencia.

Rodríguez Piñero-Royo, M. (2012). La enseñanza del Derecho del Trabajo en los Grados de estudios laborales. En J. Cruz Villalón, J. y M. Rodríguez-Piñero Royo, La enseñanza del Derecho del Trabajo y de la Seguridad Social en los nuevos Planes de Estudio. Murcia. Laborum.

Rivero, A. y Porlán, R. (2017). La evaluación en la enseñanza universitaria. En R. Porlán (Coord.), Enseñanza Universitaria, Cómo mejorarla. Madrid: Morata.

Mora, F. (2017). Neuroeducación: Sólo se puede aprender aquello que se ama. Madrid: Alianza Editorial.

Fernández Enguita, M. (2018). El aula está muy lejos de la marcha del cambio social, del mundo real (entrevista). Recuperado de: https://www.diariodesevilla.es/ entrevistas.

Jornadas de Formación e Innovación Docente del Profesorado | № 1 (2018) Esta obra se distribuye con la licencia Creative Commons 